\title{
SUMS OF DISTANCES BETWEEN POINTS ON A SPHERE. II
}

\author{
KENNETH B. STOLARSKY
}

\begin{abstract}
Given $N$ points on a unit sphere in Euclidean $m$ space, $m \geqq 2$, we show that the sum of all distances which they determine plus their discrepancy is a constant. As applications we obtain (i) an upper bound for the sum of the distances which for $m \geqq 5$ is smaller than any previously known and (ii) the existence of $N$ point distributions with small discrepancy. We make use of W. M. Schmidt's work on the discrepancy of spherical caps.
\end{abstract}

1. Introduction. For $m \geqq 2$ let $d$ be a function on $U \times U$ where $U=U^{m}$, the surface of the unit sphere of $m$ dimensional Euclidean space $E^{m}$. Let $M_{p}$ be a sequence of $N$ points $p_{1}, \cdots, p_{N} \in U^{m}$. Define

$$
S\left(N, m, M_{p}\right)=S\left(d ; N, m, M_{p}\right)=\sum_{i<j} d\left(p_{i}, p_{j}\right)
$$

and

$$
S(N, m)=S(d ; N, m)=\max S\left(d ; N, m, M_{p}\right)
$$

where the maximum is taken over all sequences $M_{p}$. We wish to obtain estimates for $S(N, m)$. Our main result, Theorem 2 of $\$ 2$, shows in a very exact sense that for a certain class of functions $d$, including the usual Euclidean distance $d(p, q)=|p-q|$, the quantity $S\left(N, m, M_{p}\right)$ is large or small depending upon whether $D\left(M_{p}\right)$, the discrepancy of $M_{p}$, is small or large. Since W. M. Schmidt [9] has obtained very good results on the discrepancy of point distributions on $U$, we can obtain (see Theorem 1 below) estimates on $S(N, m)$ which are far better than any hitherto known. Earlier results can be found, inter alia, in [1], [2], [3, p. 261, Remark 1], [4], [5], [8, pp. 36-38], and [12]; however, this paper can be read independently of these. Also, by applying Lemma 2.4 of [1] we can show (see Theorem 3 of $\$ 4$ ) that there exist finite sequences $M_{p}$ having small discrepancy. A precise definition of the discrepancy $D\left(M_{p}\right)$ is given after the statement of Theorem 2.

If $d_{0}$ is the great circle metric then $S\left(d_{0} ; N, m\right)=(\pi / 4) N^{2}$ for $N$ even (see

Presented to the Society, December 18, 1972; received by the editors February 2, 1973. AMS (MOS) subject classifications (1970). Primary 52A25, 52A40.

(c) American Mathematical Society 1973 
[7] and [11]; [7] also discusses the case $N$ odd). For $q_{1}, q_{2} \in U$ define

$$
\begin{aligned}
d_{1}\left(q_{1}, q_{2}\right) & =\sigma(U)^{-1} \int\left|d_{0}\left(q_{1}, p\right)-d_{0}\left(q_{2}, p\right)\right| d \sigma(p) \\
& \leqq d_{0}\left(q_{1}, q_{2}\right) \leqq(\pi / 2)\left|q_{1}-q_{2}\right|
\end{aligned}
$$

where $d \sigma(p)$ is the element of surface area on $U$, and the total surface area of $U$ is denoted by $\sigma(U)$. Clearly $d_{1}$ is a metric. Now (and henceforth) we let $c(m), c_{1}(m), c_{i}(m, \varepsilon)$, etc., denote positive constants depending on the indicated parameters. Here $c(m)$ and $c_{1}(m)$ equal the coefficient of $N^{2}$ in (2.4), for the appropriate $\rho$.

THEOREM 1. For $\varepsilon>0$ and $m \geqq 3$ we have

$$
\begin{aligned}
c_{1}(m) N^{2}-c_{2}(m) N^{1-1 /(m-1)} & <S\left(d_{1} ; N, m\right) \\
& <c_{1}(m) N^{2}-c_{3}(m, \varepsilon) N^{\theta_{1}(m)-\varepsilon}
\end{aligned}
$$

where $\theta_{1}(m)=\left(m^{2}-4 m+2\right)(m-1)^{-2}$. Similarly, when $d$ is the Euclidean metric and $m \geqq 3$ we have

$$
\begin{aligned}
c(m) N^{2}-c_{4}(m) N^{1-1 /(m-1)} & <S(d ; N, m) \\
& <c(m) N^{2}-c_{5}(m, \varepsilon) N^{\theta(m)-\varepsilon}
\end{aligned}
$$

where $\theta(m)=\left(m^{2}-5 m+2\right)(m-1)^{-2}$.

The older results, when applied to $d$, yield at best

$$
S(d ; N, m)<c(m) N^{2}-c^{\prime}(m)
$$

for some constant $c^{\prime}(m)>0$. In fact, aside from [1], they yield results weaker than (1.6). Thus for $m \geqq 5$ the result of (1.5) is stronger than any previously known. It suggests the following question: is there a positive constant $h(m)$ such that

$$
S(N, m)=c(m) N^{2}-h(m) N^{1-1 /(m-1)}+o\left(N^{1-1 /(m-1)}\right) ?
$$

2. The main result. We let $p_{0} \in U$ denote the vector $(1,0, \cdots, 0)$ and we denote the inner product of vectors $p, q \in U$ by $p \cdot q$. Note that $p \cdot \tau q=\tau^{-1} p \cdot q$ for any orthogonal transformation $\tau \in S O=S O(m)$, the special orthogonal group acting on $U^{m}$. We let $\int \cdots d \tau$ denote a normalized Haar integral over this group. For integrals on the real line we let $\int_{a, b}$ denote $\int_{a}^{b}$ unless $b<a$, in which case it shall denote $\int_{b}^{a}$.

Definition 2.1. For $p_{1}, p_{2} \in U$ and a function $g=g(x)$ integrable in $[0,1]$ we define

$$
\rho\left(p_{1}, p_{2}\right)=\rho\left(g ; p_{1}, p_{2}\right)=\iint_{\tau p_{1} \cdot p_{0}, \tau p_{2} \cdot p_{0}} g(x) d x d \tau .
$$


Clearly $\rho$ is independent of $p_{0}$, and for $\tau_{1} \in S O$ we have

$$
\rho\left(\tau_{1} p_{1}, \tau_{1} p_{2}\right)=\rho\left(p_{1}, p_{2}\right) .
$$

Note that $\rho$ is a metric if $g(x)>0$; however, our proof of Theorem 2 does not require this hypothesis. We call $g$ the kernel of $\rho$. If $g(x)=1$ then $\rho\left(p_{1}, p_{2}\right)$ is a constant multiple of the Euclidean distance $\left|p_{1}-p_{2}\right|$. The metric $d_{1}$ of $\S 1$ has kernel $\left(1-x^{2}\right)^{-1 / 2}$. It is sometimes useful to relax the restriction on $g$ to integrability on closed subintervals of the open interval $(0,1)$ and consider $\rho$ to be defined whenever the inner integral is integrable over $S O$. In particular this permits the kernel $\left(1-x^{2}\right)^{-1}$.

Definition 2.2. Let $\sigma(x)=\left\{p \in U \mid p \cdot p_{0} \leqq x\right\}$. We also denote the surface area of this set by the same symbol. Thus

$$
\sigma(x)=\int_{p \cdot p_{0} \leqq x} d \sigma(p) \text { and } \sigma(1)=\sigma(U) .
$$

Set $\sigma^{*}(x)=\sigma(x) / \sigma(U)$. Next, let $f_{p}=f\left(M_{p}, \tau, x\right)=\left|M_{p} \cap \tau \sigma(x)\right|$ where $|T|$ denotes the cardinality of the set $T$. Thus $f_{p}$ is the number of points of $M_{p}$ which lie in a certain spherical cap congruent to $\sigma(x)$.

THEOREM 2.

$$
\begin{aligned}
S\left(\rho ; N, m, M_{p}\right)+\int_{-1}^{1} g(x) \int & \left(f\left(M_{p}, \tau, x\right)-N \sigma^{*}(x)\right)^{2} d \tau d x \\
= & N^{2} \cdot 2^{-1} \sigma(U)^{-2} \iint \rho(p, q) d \sigma(p) d \sigma(q) .
\end{aligned}
$$

This is our main result. The second term on the left of (2.4) clearly measures the discrepancy of $M_{p}$; i.e. the extent to which it deviates from a uniform distribution. We denote it by $D\left(M_{p}\right)$, and call it the "discrepancy of $M_{p}$ with respect to the weight (or kernel) $g(x)$ ". Theorem B of [9] shows that, for $\varepsilon>0$,

$$
D\left(M_{p}\right) \gg N^{1-1 /(m-1)-\varepsilon}
$$

for any $M_{p}$ when $g(x)=\left(1-x^{2}\right)^{-1}$; the implied constant depends on $\varepsilon$. Theorem 2 is perhaps best appreciated as an invariance principle: the sum of all distances determined by $N$ points plus their discrepancy is constant.

3. The proof. First we prove a useful identity, various forms of which have already appeared explicitly or implicitly in the literature; see [1], [2], [6, p. 196, Theorem 3.1], [10], and [12].

LemmA. If $p_{i}$ and $q_{j}$ are real numbers for $1 \leqq i \leqq u$ and $1 \leqq j \leqq v$ with $p_{1} \leqq \cdots \leqq p_{u}$ and $q_{1} \leqq \cdots \leqq q_{v}$ then

$$
\sum_{i, j} \int_{p_{i}, a_{j}}-\sum_{i<j} \int_{p_{i}, p_{j}}-\sum_{i<j} \int_{q_{i}, q_{j}}=\int_{-\infty}^{\infty} g(x) G(G-(u-v)) d x
$$


where $\int_{a, b}=\int_{a, b} g(x) d x$ and

$$
G=\sum_{p_{j} \leqq x} 1-\sum_{a_{j} \leqq x} 1 .
$$

Note. If $u=v$ and $g(x)=1$ we have

$$
\int_{-\infty}^{\infty} G^{2} d x \geqq \int_{-\infty}^{\infty}|G| d x=\sum_{i=1}^{u}\left|p_{i}-q_{i}\right|
$$

and (3.1) becomes the inequality of Lemma 2.1 in Alexander's paper [1].

Proof. Let $x$ be a real number distinct from any $p_{i}$ or $q_{j}$. Let $s$ be the number of $p_{i}$ to the left of $x$ and $t$ the number of $q_{j}$ to the left of $x$. From the identity

$$
s(v-t)+t(u-s)-s(u-s)-t(v-t)=(s-t)((s-t)-(u-v))
$$

we see that $g(x)$ occurs on the left of (3.1) the same net number of times as it occurs on the right. This proves the Lemma.

We begin our proof of Theorem 2 by letting $u=v=N$ and letting $M_{p}$ and $M_{q}$ be the sequences $p_{1}, \cdots, p_{N}$ and $q_{1}, \cdots, q_{N}$ respectively. We let $\phi_{1}, \phi_{2}$, and $\tau$ be elements of $S O$ and

$$
\sum=\sum_{i, j} \rho\left(\phi_{1} p_{i}, \phi_{2} q_{j}\right)
$$

If $R$ denotes the right side of (2.4) then

$$
2 R=\iint \sum d \phi_{1} d \phi_{2}
$$

We now recall (2.1), apply the above lemma to $\sum$ with $p_{i}$ and $q_{j}$ replaced by $\tau \phi_{1} p_{i} \cdot p_{0}$ and $\tau \phi_{2} q_{j} \cdot p_{0}$ respectively, and then apply (2.2). This, and an integration over $\tau$, yields

$$
\begin{aligned}
\sum= & \sum_{i<j} \rho\left(p_{i}, p_{j}\right)+\sum_{i<j} \rho\left(q_{i}, q_{j}\right) \\
& +\iint_{-1}^{1} g(x)\left(\sum_{\tau \phi_{1} p_{i} \cdot p_{0} \leqq x} 1-\sum_{\tau \phi_{2} q_{i} \cdot p_{0} \leqq x} 1\right)^{2} d x d \tau .
\end{aligned}
$$

Upon inserting (3.4) into (3.3) we obtain

$$
\begin{aligned}
2 R= & S\left(N, m, M_{p}\right)+S\left(N, m, M_{q}\right) \\
& +\int_{-1}^{1} \iint g(x)\left(f\left(M_{p}, \phi_{1}, x\right)-f\left(M_{q}, \phi_{2}, x\right)\right)^{2} d \phi_{1} d \phi_{2} d x .
\end{aligned}
$$

Now clearly

$$
\int\left(f_{p}-N^{*}\right) d \phi_{1}=\int\left(f\left(M_{p}, \phi_{1}, x\right)-N \sigma^{*}(x)\right) d \phi_{1}=0
$$


since $N^{*}=N \sigma^{*}(x)$ is just the expected value of $f_{p}=f\left(M_{p}, \phi_{1}, x\right)$. Since

$$
\left(f_{p}-f_{q}\right)^{2}=\left(f_{p}-N^{*}\right)^{2}-2\left(f_{p}-N^{*}\right)\left(f_{q}-N^{*}\right)+\left(f_{q}-N^{*}\right)^{2}
$$

and the integral of the middle term on the right of (3.7) is zero, the right side of (3.5) is the sum of the discrepancies of $M_{p}$ and $M_{q}$ with respect to $g(x)$. The proof is completed by setting $p_{i}=q_{i}$ for $1 \leqq i \leqq N$ and dividing both sides of (3.5) by 2 .

Note. One could obtain a result having a more general appearance than (2.4) by using the full strength of the lemma rather than the special case $u=v$. A comparison of this result with (2.4) yields the identity

$$
\int_{-1}^{1} g(x) \sigma(x)(\sigma(U)-\sigma(x)) d x=\frac{1}{2} \iint \rho(p, q) d \sigma(p) d \sigma(q) .
$$

4. Some applications. We first introduce some notation from [9]. Let $\mu$ be the normalized Lebesgue measure on $U$; thus $\mu(U)=1$. Let $C=C(r, p)$ be the spherical cap consisting of all points whose spherical distance from $p \in U$ is at most $r$. Note that $0 \leqq r \leqq \pi$. Let $\Delta=\Delta(r, p)=N \mu(C)-v(C)$ where $v(C)$ denotes the number of $p_{1}, \cdots, p_{N}$ which lie in $C$. Let $E(r)=\int_{U} \Delta^{2} d \mu(p)$. Schmidt's Theorem B is given on p. 59 of [9]. By replacing his $n$ and $\delta$ with $m-1$ and $\pi / 2$ respectively we obtain the

THEOREM. If $m \geqq 3, \varepsilon>0$, and $N>\varepsilon$ then

$$
\int_{0}^{\pi / 2} r^{-1} E(r) d r \geqq c_{6}(m, \varepsilon) N^{1-1 /(m-1)-\varepsilon} .
$$

For later use we note the trivial estimate

$$
E(r) \leqq c_{7}(m) N^{2} r^{m-1} \text {. }
$$

(This is Lemma 4, p. 67 of [9]; set $s=r$ and note that Schmidt's $E(r, r)$ is our $E(r)$.) We now obtain a result which shows that (4.1) is not too far from best possible in its dependence on $N$. For $m \geqq 3$ we have

THEOREM 3. There are points $p_{1}, \cdots, p_{N}$ on $U$ such that

$$
\int_{0}^{\pi / 2} r^{-1} E(r) d r \leqq c_{8}(m) N^{1-1 /(m-1)} .
$$

To prove this we need the following special case of a result of Alexander (Lemma 2.4 of [1]).

LEMMA. Let $\rho\left(p_{1}, p_{2}\right)$ be a nonnegative function on $U \times U$ and let $\mu$ be a Borel measure for which $\mu(U)=1$. Let $P=\left\{A_{1}, \cdots, A_{N}\right\}$ be a collection of Borel subsets of $U$ such that $\mu\left(A_{i} \cap A_{j}\right)=\delta_{i j} N^{-1}$ where $\delta_{i j}$ is 1 if $i=j$ and 0 
otherwise. Then

$$
N^{2} \int_{U} \int_{U} \rho(p, q) d \mu(p) d \mu(q)-\sum_{i=1}^{N} \rho\left(A_{i}\right) \leqq 2 S(\rho ; N, m)
$$

where $\rho\left(A_{i}\right)$ is the "diameter" of $A_{i}$; i.e. $\rho\left(A_{i}\right)=\sup \rho(p, q)$ for $p, q \in A_{i}$ (Alexander has $\left|p_{1}-p_{2}\right|$ in place of $\rho\left(p_{1}, p_{2}\right)$ but his proof required only the nonnegativity of this function).

We now choose $M_{p}$ so that $S\left(\rho ; N, m, M_{p}\right)=S(\rho ; N, m)$. It follows from (2.4) and (4.4) that

$$
\int_{-1}^{1} g(x) \int\left(f\left(M_{p}, \tau, x\right)-N \sigma^{*}(x)\right)^{2} d \tau d x \leqq c_{9}(m) N \inf _{P} \max _{i} \rho\left(A_{i}\right),
$$

a result of interest in itself.

To prove Theorem 3 let $g(x)=\left(1-x^{2}\right)^{-1}$ and set $p_{1} \cdot p=\cos \theta_{1}$ and $p_{2} \cdot p=\cos \theta_{2}$ where $\theta_{1}$ and $\theta_{2}$ are the great circle distances between $p_{1}, p$ and $p_{2}, p$ respectively. Then

$$
\begin{aligned}
\rho & =\rho\left(p_{1}, p_{2}\right)=c_{9}(m) \iint_{p_{1} \cdot p_{12} \cdot p} g(x) d x d \sigma(p) \\
& =c_{10}(m) \int\left|\log \tan \frac{1}{2} \theta_{1}-\log \tan \frac{1}{2} \theta_{2}\right| d \sigma(p) .
\end{aligned}
$$

It is easy to see that this integral is finite since $\int\left|\log \tan \frac{1}{2} \theta_{1}\right| d \sigma(p)$ is finite. Now

$$
\begin{aligned}
\left|p_{1}-p_{2}\right|^{-1} \rho\left(p_{1}, p_{2}\right) & =c_{10}(m) \int\left\{\left|\theta_{1}-\theta_{2}\right|^{-1}\left(\log \tan \frac{1}{2} \theta_{1}-\log \tan \frac{1}{2} \theta_{2}\right)\right\} \\
& \quad \cdot\left\{\left|\theta_{1}-\theta_{2}\right| \cdot\left|p_{1}-p_{2}\right|^{-1}\right\} d \sigma(p) \\
& \leqq c_{11}(m) \int\left|\theta_{1}-\theta_{2}\right|^{-1}\left(\log \tan \frac{1}{2} \theta_{1}-\log \tan \frac{1}{2} \theta_{2}\right) d \sigma(p) \\
& \equiv \int_{U} .
\end{aligned}
$$

We will show that $\int_{U}$ remains bounded as $p_{2} \rightarrow p_{1}$. Let the great circle distance between $p_{1}$ and $p_{2}$ be $\gamma \pi / 10$ where $\gamma$ is a small positive parameter. Let $B_{1}=B_{1}(\gamma)$ be the set of points $p \in U$ such that $\gamma \pi \leqq \theta_{i} \leqq(1-\gamma) \pi$ for $i=1,2$. Then $B_{2}$, the complement of $B_{1}$, consists of two components, say $B_{3}$ and $B_{4}$. The estimates we need for the integrals over $B_{3}$ and $B_{4}$ will be essentially identical, so we give details only for $B_{4}$ which we take to be the component for which $\theta_{1}, \theta_{2} \leqq 2 \gamma \pi$. Write $B_{4}=B_{5} \cup B_{6}$ where $B_{5}, B_{6}$ are disjoint and $B_{5}$ consists of those points of $B_{4}$ for which $\theta_{1} \leqq 2 \theta_{2}$. Then

$$
\int_{U}=\int_{B_{1}}+\int_{B_{3}}+\int_{B_{5}}+\int_{B_{6}} \ll \int_{B_{1}}+\int_{B_{5}}+\int_{B_{6}} \text {. }
$$


The following two estimates make use of the fact that $d \sigma(p) \ll \theta_{i}^{m-2} d \theta_{i}$, and require $m \geqq 3$ :

and

$$
\int_{B_{5}} \ll \int_{B_{5}}\left|\sin \left(\min \left(\theta_{1}, \theta_{2}\right)\right)\right|^{-1} d \sigma(p) \ll \int_{B_{5}} \theta_{1}^{-1} d \sigma(p) \ll \int_{0}^{\gamma \pi} d \theta=\gamma \pi
$$

Also,

$$
\int_{B_{6}} \ll \int_{B_{6}}\left|\theta_{2}^{-1} \log \theta_{2}\right| d \sigma(p) \ll \int_{0}^{\gamma \pi}|\log \theta| d \theta \ll \gamma|\log \gamma|
$$

$$
\int_{B_{1}} \ll \int_{B_{1}}\left(\left|\sin \theta_{1}\right|^{-1}+\left|\sin \theta_{2}\right|^{-1}\right) d \sigma(p) \ll 2 \int_{U}\left|\sin \theta_{1}\right|^{-1} d \sigma(p)<\infty .
$$

Thus $\rho\left(p_{1}, p_{2}\right) \ll\left|p_{1}-p_{2}\right|$. Now extend the integral on the left of (4.5) only to 0 , and make the change of variable $x=-\cos r$. This yields

$$
\int_{0}^{\pi / 2} r^{-1} E(r) d r \leqq c_{12}(m) N \inf _{P} \max _{i} \rho\left(A_{i}\right) .
$$

Now clearly one can choose the $A_{i}$ so that their Euclidean diameters are $\gg \ll N^{-1 /(m-1)}$ for $1 \leqq i \leqq N$. But $\rho\left(p_{1}, p_{2}\right) \ll\left|p_{1}-p_{2}\right|$ so the result follows.

An immediate consequence of Theorem 1 and the above is that if $\rho$ has kernel $\left(1-x^{2}\right)^{-1}$ then

$$
\begin{aligned}
c_{13}(m) N^{2}-c_{14}(m) N^{1-1 /(m-1)} & <S(\rho ; N, m) \\
& <c_{13}(m) N^{2}-c_{16}(m, \varepsilon) N^{1-1 /(m-1)-\varepsilon} .
\end{aligned}
$$

We now prove Theorem 1 . The left-hand inequalities are deduced from (4.4) as in the proof of Theorem 3. For the right-hand inequality of (1.5) we set $g(x) \equiv 1$ and note that

$$
\begin{aligned}
D\left(M_{p}\right) & \geqq c_{17}(m) \int_{-1}^{0} E\left(\cos ^{-1}|x|\right) d x=c_{17}(m) \int_{0}^{\pi / 2} \sin r E(r) d r \\
& \geqq c_{18}(m) \int_{0}^{\pi / 2} r E(r) d r \geqq c_{18}(m) N^{-\alpha} \int_{N^{-\alpha}}^{\pi / 2} E(r) d r
\end{aligned}
$$

for any $\alpha>0$. From (4.1) and (4.2) we obtain

$$
c_{7}(m) N^{2} \int_{0}^{N^{-\alpha}} r^{m-2} d r+N^{\alpha} \int_{N^{-\alpha}}^{\pi / 2} E(r) d r \geqq c_{6}(m, \varepsilon) N^{1-1 /(m-1)-\varepsilon} .
$$

Set $\alpha=1 /(m-1)+1 /(m-1)^{2}+2 \varepsilon /(m-1)$. Then the term on the extreme left of (4.8) has lower order of magnitude than the term on the extreme right, so it follows that

$$
D\left(M_{p}\right) \geqq c_{19}(m, \varepsilon) N^{1-(3+4 \varepsilon) /(m-1)-2 /(m-1)^{2}-\varepsilon} .
$$


To prove (1.4) we note that in this case

so

$$
D\left(M_{p}\right) \geqq c_{20}(m) \int_{N^{-\alpha}}^{\pi / 2} E(r) d r,
$$

$$
D\left(M_{p}\right) \geqq c_{21}(m, \varepsilon) N^{1-(2+2 \varepsilon) /(m-1)-1 /(m-1)^{2}-\varepsilon} .
$$

This completes the proof of Theorem 1 .

I would like to thank J. Ralph Alexander for helpful conversations concerning this work, and also for making available to me a preprint of [1].

\section{REFERENCES}

1. J. Ralph Alexander, On the set of distances determined by $n$ points on the 2-sphere, Acta Math. Acad. Sci. Hungar. 23 (1972), 443-448.

2. J. Ralph Alexander and K. B. Stolarsky, Extremal problems of distance geometry related to energy integrals, Trans. Amer. Math. Soc. (to appear).

3. G. Björck, Distributions of positive mass which maximize a certain generalized energy integral, Ark. Mat. 3 (1956), 255-269. MR 17, 1198.

4. L. Fejes Toth, On the sum of distances determined by a pointset, Acta Math. Acad. Sci. Hungar. 7 (1956), 397-401. MR 21 \#5937.

5. E. Hille, Some geometric extremal problems, J. Austral. Math. Soc. 6 (1966), 122-128. MR 33 \#6507.

6. J. B. Kelly, Metric inequalities and symmetric differences, Inequalities, II (Proc. Second Sympos., U.S. Air Force Acad., Colo., 1967), Academic Press, New York, 1970, pp. 193-212. MR 41 \#9192.

7. F. Nielson, Om summen af afstandene mellem n punkter $\mathrm{pa}^{\circ}$ en Kugleflade, Nordisk Mat. Tidskr. 13 (1965), 45-50.

8. G. Pólya and G. Szegö, Über den transfiniten Durchmesser (Kapazitätskonstante) von ebenen und räumlichen Punktmengen, J. Reine Angew. Math. 165 (1931), 4-49.

9. W. M. Schmidt, Irregularities of distribution. IV, Invent. Math. 7 (1969), 55-82. MR 39 \#6838.

10. I. J. Schoenberg, On certain metric spaces arising from Euclidean spaces by change of metric and their embedding in Hilbert space, Ann. of Math. 38 (1937), 787-793.

11. G. Sperling, Lösung einer elementargeometrischen Frage von Fejes Tóth, Arch. Math. 11 (1960), 69-71. MR 22 \#2935.

12. K. B. Stolarsky, Sums of distances between points on a sphere, Proc. Amer. Math. Soc. 35 (1972), 547-549.

Department of Mathematics, University of Illinois, Urbana, Illinois 61801 